\title{
Role of Bisection Method
}

\author{
Chitra Solanki \\ DIT University \\ Dehradun, India
}

\author{
Pragati Thapliyal \\ DIT University \\ Dehradun, India
}

\author{
Komal Tomar \\ DIT University \\ Dehradun, India
}

\begin{abstract}
The bisection method is the basic method of finding a root. As iterations are conducted, the interval gets halved. So method is guaranteed to converge to a root of "f" if " $\mathrm{f}$ " is a continuous function at an interval [a,b] and $\mathrm{f}(\mathrm{a})$ and $\mathrm{f}(\mathrm{b})$ should have opposite sign. In this paper we have explained the role of bisection method in computer science research. we also introduced a new method which is a combination of bisection and other methods to prove that with the help of bisection method we can also develop new methods. It is observed that scientists and engineers are often faced with the task of finding out the roots of equations and the basic method is bisection method but it is comparatively slow. We can use this new method to solve these problems and to improve the speed.
\end{abstract}

Key words: continous, absolute error, Iteration, convergence, Newton-Raphson method, Regular- Falsi method

\section{Introduction}

Traditional iterative schemes such as Newton's method and related classes of algorithms [3,4] often fail to converge to a specific periodic orbit since their convergence is almost independent of the initial guess. Moreover, these methods are affected by the imprecision the mapping evaluations. It may also happen that these methods fail due to the nonexistence of derivatives or poorly behaved partial derivatives [3,4]. Recently, this method has been applied successfully to various difficult problems; see, for example, [7-11]. One of the first numerical methods developed to find the root of a nonlinear equation $f(x)=0$ was the bisection method (also called binary-search method)[1]. Since the method is based on finding the root between two points, the method falls under the category of bracketing methods. Since the root is bracketed between two points, $x_{\ell}$ and $x_{u}$, one can find the mid-point, $x_{m}$ between $x_{\ell}$ and $x_{u}$. This gives us two new intervals

\section{THE GRAPHICAL DISCRIPTION-:}

What is the bisection method and what is it based on? One of the first numerical methods developed to find the root of a nonlinear equation $f(x)=0$ was the bisection method (also called binary-search method). The method is based on the following theorem. [1]

\section{What is the use of bisection method :}

- It is used in computer science research to analyze safeguard zero finding methods

- It is simplest of other all methods

- We can safeguard bisection to detect cases where we don't have any roots

\section{Theorem}

An equation $f(x)=0$, where $f(x)$ is a real continuous function, has at least one root between $x_{\ell}$ and $x_{u}$ if $f\left(x_{\ell}\right) f\left(x_{u}\right)<0$ (See Figure 1).

Note that if $f\left(x_{\ell}\right) f\left(x_{u}\right)>0$, there may or may not be any root between $x_{\ell}$ and $x_{u}$ (Figures 2 and 3). If $f\left(x_{\ell}\right) f\left(x_{u}\right)<0$, then there may be more than one root between $x_{\ell}$ and $x_{u}$ (Figure 4). So the theorem only guarantees one root between $x_{\ell}$ and $x_{u}$.

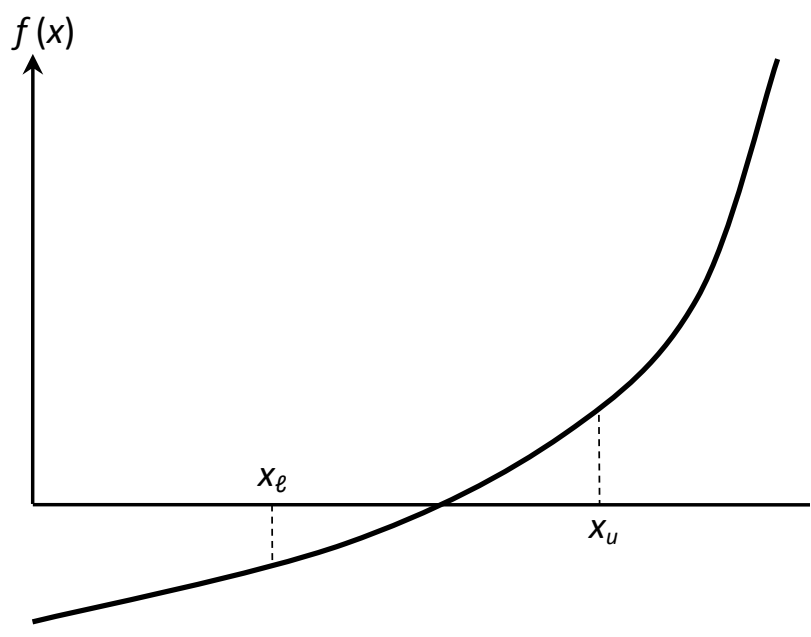

Figure 1 At least one root exists between the two points if the function is real, continuous, and changes sign. 


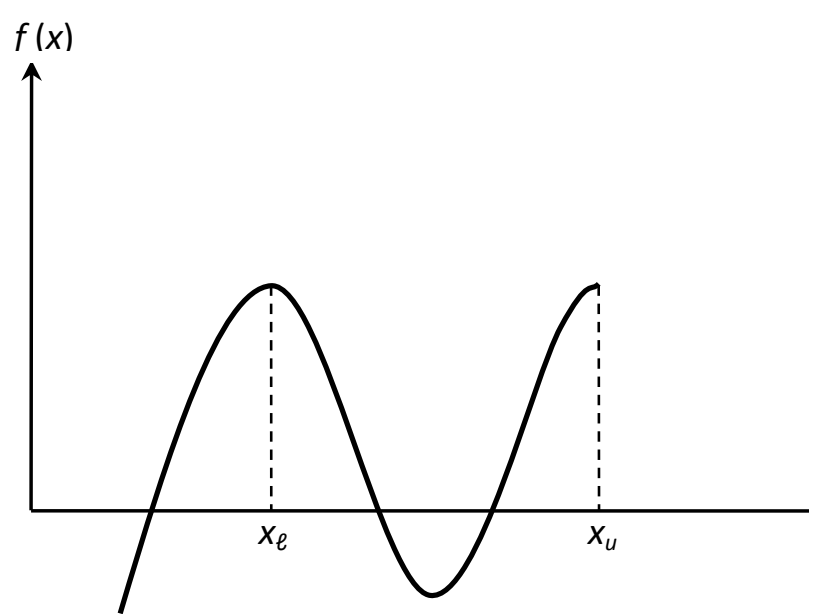

Figure 2 If the function $f(x)$ does not change sign between the two points, roots of the equation $f(x)=0$ may still exist between the two points.

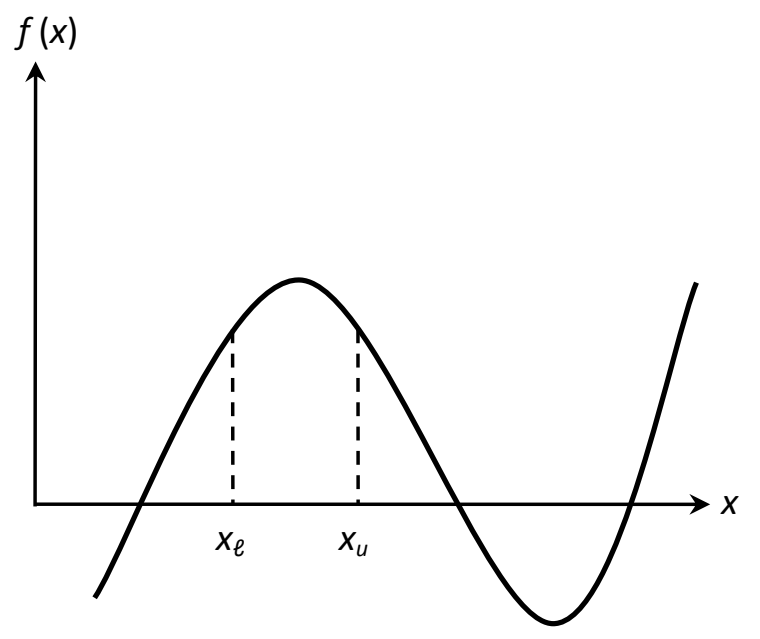

Figure 3 If the function $f(x)$ does not change sign between two points, there may not be any roots for the equation $f(x)=0$ between the two points.

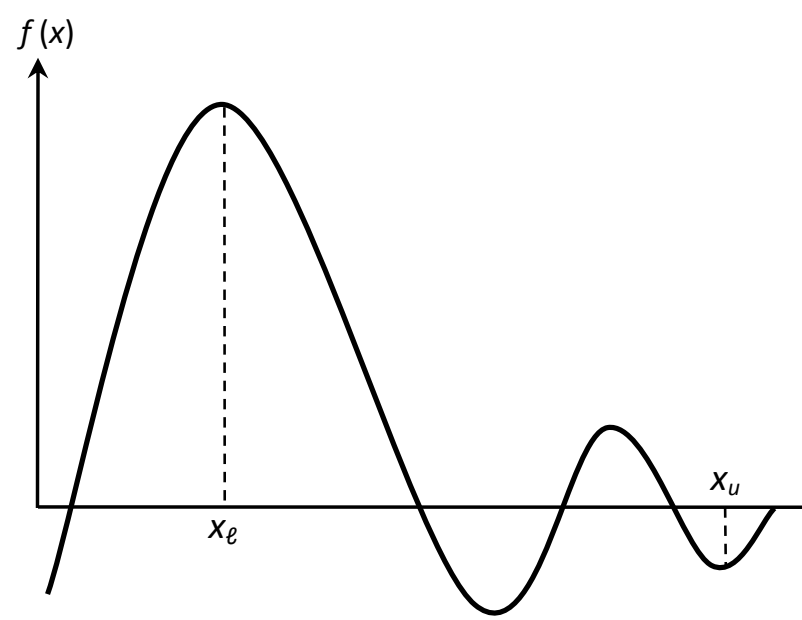

Figure 4 If the function $f(x)$ changes sign between the two points, more than one root for the equation $f(x)=0$ may exist between the two points.

3. PROBLEM DESCRIPTION:- The bisection method guarantees a root (or singularity) and is used to limit the changes in position estimated by the Newton-Raphson method when the linear assumption is poor. However, Newton-Raphson steps are taken in the nearly linear regime to speed convergence.

In other words, if we know that we have a root bracketed between our two bounding points, we first consider the Newton-Raphson step. If that would predict a next point that is outside of our bracketed range, then we do a bisection step instead by choosing the midpoint of the range to be the next point. We then evaluate the function at the next point and, depending on the sign of that evaluation, replace one of the bounding points with the new point. This keeps the root bracketed, while allowing us to benefit from the speed of Newton-Raphson. Wrong assumption of Newton-Raphson method can increase no. of iterations.

An improved root finding scheme is to combine the BISECTION and REGULAR-FALSI methods.It is relatively faster then bisection method.

\section{RELATED WORK:-}

we first analyzed some of the conventional root finding methods and their limitations. Bisection always converges but is slow. Newton has quadratic convergence but may fail in some of the cases. Secant is a good alternative to Newton but it oscillates in some of the cases and fails to converge.

- It is explained that it is important that we safeguard bisection to detect cases where 
we don't have any roots. The question of guessing the bound is more intuitive.

- The other method like Newton's method have a disadvantage that higher order roots can cause convergence to be slow, and the sequence may take undesirable jumps between roots or take a very large step upon encountering an reflection point. One case where it fails is when derivative of function $f(x)$ is either zero or infinite then it fails to converge.

- We have proposed a new method by combining Bisection method with other methods. So, that we can find roots as well as the method can be fast in solving.

- The multidimensional bisection method allows to solve constrained minimization problem when the feasible region is $\mathrm{n}$ dimensional simplex. This method does not require a differentiability of function and is guaranteed to converge to the minimize for the class of strictly unimodal function[12]

\section{PROPOSED-METHOD}

$$
x_{i+1=} 3 x_{i-1} f\left(x_{i}\right)-x_{i-1} f\left(x_{i-1}\right)+x_{i} f\left(x_{i}\right)-3 x_{i} f\left(x_{i-1}\right) / 4\left[f\left(x_{i}\right)-f\left(x_{i-1}\right)\right]
$$

\section{Algorithm for this new method:}

The steps to apply the new method to find the root of the equation Choose $\mathrm{x}_{\mathrm{i}-1}$ and $\mathrm{x}_{\mathrm{i}}$ as two guesses for the root such that $\mathrm{f}\left(\mathrm{x}_{\mathrm{i}}\right) \mathrm{f}\left(\mathrm{x}_{\mathrm{i}-1}\right)<0$, or in other words, $f(x)$ changes sign between $x_{i-1}$ and $x_{i}$.

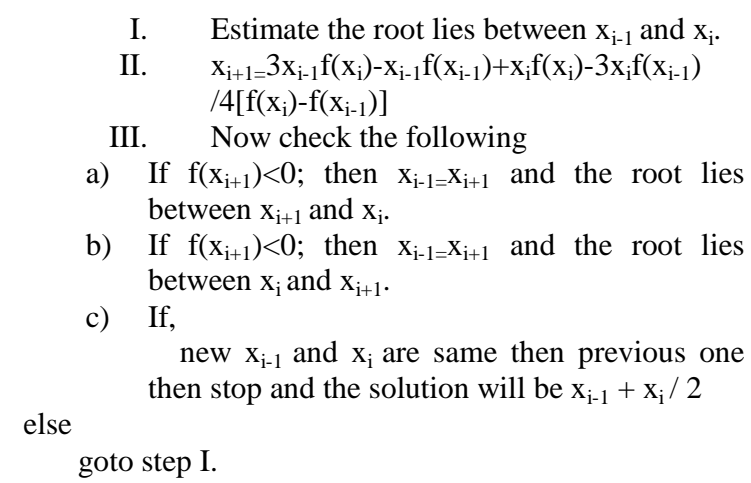

\section{Comparisons table of new method with existing methods:-}

For a given problem $\mathbf{f}(\mathbf{x})=\mathbf{x}^{3}-7$.[2]The comparison is done between four methods the new method is as faster as NewtonRaphson method and Regular -Falsi method and also accurate as we don't take any guess
Table 1 Comparison

\begin{tabular}{|l|l|l|}
\hline S.No. & Method name & No. of iterations \\
\hline $\mathbf{1}$ & $\begin{array}{l}\text { BISECTION } \\
\text { METHOD }\end{array}$ & 14 \\
\hline $\mathbf{2}$ & $\begin{array}{l}\text { REGULAR-FALSI } \\
\text { METHOD }\end{array}$ & 5 \\
\hline $\mathbf{3}$ & $\begin{array}{l}\text { NEWTON } \\
\text { RAPHSON } \\
\text { METHOD }\end{array}$ & 5 \\
\hline $\mathbf{4}$ & NEW METHOD & 6 \\
\hline
\end{tabular}

\section{CONCLUSION:}

Bisection method is the safest and it always converges. The bisection method is the simplest of all other methods and is guaranteed to converge for a continuous function. It is always possible to find the number of steps required for a given accuracy.and the new methods can also be developed from bisection method and bisection method plays a very crucial role in computer science research.

\section{REFERENCES :}

[1] Chapter 03.03 Bisection Method of Solving a Nonlinear Equation

[2] Book numerical based analysis from DITU library

[3] J.M. Ortega, W.C. Rheinbolt, Iterative solution of nonlinear equations in several (1970)

[4] J.E. Dennis, R.B. Schnabel, Numerical Methods for Unconstrained Optimization and Nonlinear Equations, SIAM, Philadelphia, 1996

[5] L. Drossos, O. Ragos, M.N. Vrahatis, T.C. Bountis, Phys. Rev. E 53 (1996) 1206.

[6] M.N. Vrahatis, T.C. Bountis, M. Kollmann, Inter. J. Bifurc. Chaos 6 (1996) 1425.

[7] M.N. Vrahatis, H. Isliker, T.C. Bountis, Inter. J. Bifurc. Chaos 7 (1997) 2707.

[8] H. Waalkens, J. Wiersig, H.R. Dullin, Ann. Phys. 260 (1997) 50.

[9] N. Buri'c, M. Mudrini'c, J. Phys. A: Math. Gen. 31 (1998) 1875.

[10] N. Buri'c, M. Mudrini'c, Todorovi'c, J. Phys. A: Math. Gen. 31 (1998) 7847.

[11] V.S. Kalantonis, E.A. Perdios, A.E. Perdiou, M.N. Vrahatis, Celest. Mech. Dynam. Astron. (2001), in press.

[12] A multidimensional bisection method for unconstrained minimization problem 